\title{
CLANS ON GROUP-SUPPORTING SPACES ${ }^{1}$
}

\section{J. SELDEN AND B. MADISON}

1. Introduction. By a clan we mean a compact connected semigroup with unit. We speak of a topological space $X$ supporting a group structure if there is a topological group having $X$ as its underlying space. Hudson and Mostert [7] have proven that a finite dimensional homogeneous clan is a group. There exist $[7$, p. 41] infinite dimensional homogeneous clans which are not groups; however, certain homogeneous clans (finite or infinite dimensional), namely those whose spaces will support group structures, are shown here to be groups. Thus the question, "Which clans are groups?", has a purely topological answer.

2. Preliminary results. Homomorphisms of topological groups and local groups are assumed to be continuous and open. A monomorphism is a one-to-one homomorphism, and an onto monomorphism is an isomorphism.

For details on the structure of the cohomology groups used here, we refer to a paper of $\mathrm{Hu}$ [4]. We denote the additive group of real numbers by $R$ and use only $R$ as a coefficient group. Let $H^{n}(X)$ represent the usual $n$-dimensional Alexander-Wallace-Spanier cohomology group of the space $X$. We now sketch the structure and give some properties of a cohomology group of a local group [4, p. 415].

Let $V$ be any local group $\left[8\right.$, p. 83] and $V^{\prime}$ a local subgroup of $V$ [8, p. 84]. For each integer $n>0$, an $n$-function of $V$ modulo $V^{\prime}$ is a real-valued continuous function $\phi$ defined on a subset $W^{n}$ of the space $V^{n}$ for some open neighborhood $W$ of the unit $e$ in $V$ such that

$$
\phi\left(g v_{1} h_{1}, \cdots, g v_{n} h_{n}\right)=\phi\left(v_{1}, \cdots, v_{n}\right)
$$

for each $\left(v_{1}, \cdots, v_{n}\right)$ in $W^{n}$ and arbitrary $g$ in $V^{\prime}$ and $h_{i}$ in $V^{\prime}$ $(i=1,2, \cdots, n)$ provided $g v_{i} h_{i}$ is defined and is in $W$ for each $i=1,2, \cdots, n$.

Two $n$-functions, $\phi: W^{n} \rightarrow R$, of $V$ modulo $V^{\prime}$ are said to be equivalent $(\phi \equiv \psi)$ if there exists an open neighborhood $W^{\prime \prime}$ of $e$ contained in the intersection $W \cap W^{\prime}$ such that $\phi$ agrees with $\psi$ on $W^{\prime \prime n}$. Thus

Received by the editors May 6, 1966.

1 The contribution of the second author was supported by an NSF Research Participation Award at the University of Oklahoma, an Academic Year Extension of this award, and an NSF Cooperative Fellowship and will constitute a portion of his doctoral dissertation at the University of Kentucky. 
the $n$-functions of $V$ modulo $V^{\prime}$ are divided in to disjoint equivalence classes, called the $n$-cochains of $V$ modulo $V^{\prime}$. Denote by $C^{n}\left(V, V^{\prime}\right)$ the set of all $n$-cochains of $V$ modulo $V^{\prime}$. In a straightforward manner one can define an addition on $C^{n}\left(V, V^{\prime}\right)$ so that it becomes an abelian group. In case $n=0$, set $C^{0}\left(V, V^{\prime}\right)=R$.

Define a coboundary operator $\delta: C^{n}\left(V, V^{\prime}\right) \rightarrow C^{n+1}\left(V, V^{\prime}\right)$ by the following procedure. If $c$ is in $C^{0}\left(V, V^{\prime}\right)$, let $\delta(c)=0$. For $n>0$ and $c$ in $C^{n}\left(V, V^{\prime}\right)$, choose an $n$-function, $\phi: W^{n} \rightarrow R$, of $V$ modulo $V^{\prime}$ such that $c=[\phi]$, that is, $\phi$ is an element of the equivalence class $c$. Choose an open neighborhood $W_{0}$ of $e$ in $V$ such that if $u$ and $v$ are in $W_{0}$, then $u^{-1} v$ is defined and is in $W$. Define an $(n+1)$-function $\delta \phi: W_{0}^{n+1} \rightarrow R$ by setting

$$
\begin{aligned}
\delta \phi\left(v_{1}, \cdots, v_{n+1}\right)= & \phi\left(v_{1}^{-1} v_{2}, \cdots, v_{1}^{-1} v_{n+1}\right) \\
& +\sum_{i=1}^{n+1}(-1)^{i} \phi\left(v_{1}, \cdots, v_{i-1}, v_{i+1}, \cdots, v_{n+1}\right)
\end{aligned}
$$

for each $\left(v_{1}, \cdots, v_{n+1}\right)$ in $W_{0}^{n+1}$. It can be seen that $\delta \phi$ is an $(n+1)$ function of $V$ modulo $V^{\prime}$ and that $[\delta \phi]$ does not depend on the choice of $\phi$. Then we can define $\delta(c)=[\delta \phi]$. Using the coboundary operator and $C^{n}\left(V, V^{\prime}\right)$, we construct the $n$th cohomology group of $V$ modulo $V^{\prime}$ and denote this by $H^{n}\left(V, V^{\prime}\right)$.

Let $G$ be a compact connected group, and choose $V$ to be a neighborhood of the unit $e$ in $G$. Considering $G$ as operating on itself by left translations, it follows from results of $\mathrm{Hu}$ [4] that there exists an isomorphism $k: H^{n}(G) \rightarrow H^{n}(V,\{e\})$.

Let $G$ and $L$ be compact connected groups and $f: G \rightarrow L$ an onto homomorphism. Let $W$ be an open neighborhood of the unit $e_{2}$ in $L$ and $V=f^{-1}(W)$. Let $g$ be the restriction of $f$ to $V$, that is, $g=f \mid V$. We want to define a homomorphism $g^{\#}$ from $C^{n}\left(W,\left\{e_{2}\right\}\right)$ to $C^{n}\left(V,\left\{e_{1}\right\}\right)$. If $n=0$, we let $g^{4}$ be the identity on $R$. Assume that $n>0$ and choose $c$ in $C^{n}\left(W,\left\{e_{2}\right\}\right)$. Choose an $n$-function, $\phi: W_{1}^{n} \rightarrow R$, of $W$ (modulo $\left\{e_{2}\right\}$ ) such that $c=[\phi]$. Define an $n$-function, $g^{\sharp} \phi:\left[g^{-1}\left(W_{1}\right)\right]^{n} \rightarrow R$, of $V$ by setting $g^{\#} \phi\left(v_{1}, \cdots, v_{n}\right)=\phi\left(g\left(v_{1}\right), \cdots, g\left(v_{n}\right)\right)$ for each $\left(v_{1}, \cdots, v_{n}\right)$ in $\left[g^{-1}\left(W_{1}\right)\right]^{n}$. If $\phi$ and $\psi$ are equivalent $n$-functions of $W$, then $\phi$ agrees with $\psi$ on some $U^{n}$ where $U$ is an open neighborhood of $e_{2}$. If this is the case, then $g^{\sharp} \phi$ agrees with $g^{\sharp} \psi$ on $\left[g^{-1}(U)\right]^{n}$ and this open neighborhood of $e_{1}$ has the properties necessary to insure that $g^{\sharp} \phi$ and $g^{\sharp} \psi$ are equivalent. Thus we define $g^{\sharp}(c)=\left[g^{\sharp} \phi\right]$. It is clear that $g$ is a homomorphism which commutes with the coboundary operator and hence induces a unique homomorphism $g^{*}: H^{n}\left(W,\left\{e_{2}\right\}\right)$ $\rightarrow H^{n}\left(V,\left\{e_{1}\right\}\right)$. 
Lemma 1. If $G$ and $L$ are compact connected Lie groups and $f: G \rightarrow L$ is an onto homomorphism, then for each integer $n \geqq 0, f^{*}: H^{n}(L) \rightarrow H^{n}(G)$ is a monomorphism.

Proof. Choose any open neighborhood $W$ of the unit $e^{\prime}$ in $L$ and let $V=f^{-1}(W)$. For any integer $n \geqq 0$, let $k: H^{n}(G) \rightarrow H^{n}(V,\{e\})$ ( $e$ is the unit of $G$ ) and $k^{\prime}: H^{n}(L) \rightarrow H^{n}\left(W,\left\{e^{\prime}\right\}\right.$ ) be the isomorphism described by $\mathrm{Hu}[4]$. Letting $g=f \mid V$ and $g^{*}: H^{n}\left(W,\left\{e^{\prime}\right\}\right) \rightarrow H^{n}(V,\{e\})$ be the homomorphism induced by $g$, it is straightforward to see that $g^{*} k^{\prime}=k f^{*}$. Thus to show that $f^{*}$ is a monomorphism, it suffices to show that there exist neighborhoods $V$ of the unit in $G$ and $W$ of the unit in $L$ such that $(f \mid V)^{*}$ is a monomorphism. To accomplish this we use the results and terminology of Pontrjagin [8, Chapter IX]. Let $U^{\prime}$ be any open neighborhood of $e^{\prime}$ and $U=f^{-1}\left(U^{\prime}\right)$. Considering $U$ and $U^{\prime}$ as local Lie groups, there correspond infinitesimal groups $S$ to $U$ and $S^{\prime}$ to $U^{\prime}$. Let $\phi: S \rightarrow S^{\prime}$ be the homomorphism corresponding to $f \mid U$. Denote the kernel of $\phi$ by $K . K$ is normal in $S$ and hence there exists a normal subgroup $T$ of $S$ such that $K+T=S$ and $K \cap T=\{0\}$. Define $\lambda: T \rightarrow S$ by $\lambda(x)=x$ for each $x$ in $T$. Then $\phi \lambda: T \rightarrow S^{\prime}$ is one-to-one and onto. Let $M$ be the local subgroup of $U$ corresponding to $T$ and $h: M \rightarrow U$ the homomorphism corresponding to $\lambda$. Then $(f \mid U) h$ is a local isomorphism, that is, there exist open neighborhoods $V_{1}$ of the unit in $M$ and $W$ of the unit in $U^{\prime}$ such that $\left((f \mid U) h \mid V_{1}\right): V_{1} \rightarrow W$ is an on to monomorphism. Let $V=f^{-1}(W)$ and $g=h \mid V_{1}$. Then $(f \mid V) g: V_{1} \rightarrow W$ induces

$$
((f \mid V) g)^{*}: B^{n}(W,\{e\}) \rightarrow B^{n}\left(V,\left\{e^{\prime}\right\}\right) .
$$

It is straightforward to verify that $((f \mid V) g)^{*}$ is a monomorphism and that $((f \mid V) g)^{*}=g^{*}(f \mid V)^{*}$. Therefore $(f \mid V)^{*}$ must be a monomorphism.

The referee has pointed out that a much shorter proof of Lemma 1 exists; however, the proof given here generalizes easily to a proof for Theorem 2 .

Lemma 2. Let $G$ be a compact connected Lie group of dimension $n$. If $A$ is a closed proper subset of $G$ and $i: A \rightarrow G$ is the inclusion function, then the induced homomorphism $i^{*}: H^{n}(G) \rightarrow H^{n}(A)$ is not a monomorphism.

Proof. Choose $x$ in $G \backslash A$ and an open neighborhood $V$ of $x$ which is homeomorphic to $R^{n}$ and such that $\operatorname{cl}(V) \cap A=\phi$. Set $B=\operatorname{cl}(G \backslash V)$. Let $j:(G, \phi) \rightarrow(G, B)$ and $k:(B, \phi) \rightarrow(G, \phi)$ be inclusion functions. It is known [2, p. 314] that $j^{*}$ maps $H^{n}(G, B)$ on to $H^{n}(G, \phi)$. Looking at the exact sequence 


$$
\cdots \rightarrow H^{n}(G, B)-j^{*} \rightarrow H^{n}(G, \phi)-k^{*} \rightarrow H^{n}(B, \phi) \rightarrow \cdots,
$$

one sees that $k^{*}$ is the zero function. Let $t: A \rightarrow B$ be the inclusion function. Then $k t=i$ implies that $t^{*} k^{*}=i^{*}$ and hence that $i^{*}$ is the zero function. Since $H^{n}(G) \neq\{0\}[3]$, it follows that $i^{*}: H^{n}(G) \rightarrow H^{n}(A)$ is not a monomorphism.

3. Main result. The proof of the following uses results about compact connected semigroups which can be found in a paper by A. D. Wallace [9].

THEOREM 1. If $(S, \cdot)$ is a clan and $S$ will support a group structure, then $(S, \cdot)$ is a group.

Proof. Since $S$ will support a group structure, it follows [6, pp. 172-179] that there exists an inverse system of compact connected Lie groups, $\left(G_{\alpha}, \pi_{\alpha \beta}, D\right)$, such that $\lim _{\leftarrow} G_{\alpha}=S$ and each $\pi_{\alpha \beta}$ is an on to homomorphism. Let $e$ be idempotent in the minimal ideal of $S$ and $i: e \cdot S \cdot e \rightarrow S$ be the injection function. For each $\alpha$ in $D$, let $\pi_{\alpha}: S \rightarrow G_{\alpha}$ be the projection function $h_{\alpha}=\pi_{\alpha} \mid e \cdot S \cdot e$, and $i_{\alpha}: \pi_{\alpha}(e \cdot S \cdot e) \rightarrow G_{\alpha}$ be the injection function. Clearly, we have $\pi_{\alpha} i(x)=i_{\alpha} h_{\alpha}(x)$ for each $x$ in $e \cdot S \cdot e$.

Suppose that $\pi_{\alpha}(e \cdot S \cdot e) \neq G_{\alpha}$ for some $\alpha$ in $D$. By the continuity axiom, $\left(H^{n}\left(G_{\alpha}\right), \pi_{\alpha \beta}^{*}, D\right)$ is a direct system of groups for each integer $n \geqq 0$. By Lemma 1 , each $\pi_{\alpha \beta}^{*}$ is a monomorphism and thus each $\pi_{\alpha}^{*}$ is a monomorphism. For $p$, the dimension of $G_{\alpha}$,

$$
h_{\alpha}^{*} i_{\alpha}^{*}: H^{p}\left(G_{\alpha}\right) \rightarrow H^{p}(e \cdot S \cdot e)
$$

is not a monomorphism, but

$$
i^{*} \pi_{\alpha}^{*}: H^{p}\left(G_{\alpha}\right) \rightarrow H^{p}(e \cdot S \cdot e)
$$

is a monomorphism. This is a contradiction since $h_{\alpha}^{*} i_{\alpha}^{*}=i^{*} \pi_{\alpha}^{*}$. Thus for each $\alpha$ in $D, \pi_{\alpha}(e \cdot S \cdot e)=G_{\alpha}$ and $(S, \cdot)$ is a group since $(e \cdot S \cdot e, \cdot)$ is a group.

4. Further remarks. Theorem 1 is actually a corollary to a more general theorem. We state, with only an indication of proof, this more general theorem because we believe that a much better result can be obtained.

If $H$ is a closed subgroup of a compact connected group $G$, then the left cosets of $H$ can be considered as the points of new space, called the coset space $G / H$, with the customary quotient topology. Choose an inverse system of compact connected Lie groups, $\left(G_{\alpha}, \pi_{\alpha \beta}, D\right)$ 
such that $\lim _{\leftarrow} G_{\alpha}=G$ and such that each $\pi_{\alpha \beta}$ is an on to homomorphism. Setting $H_{\alpha}=\pi_{\alpha}(H)$ and $\pi_{\alpha \beta}^{\prime}\left(g H_{\alpha}\right)=\pi_{\alpha \beta}(g) H_{\beta}$, it can be seen [1] that $\left(G_{\alpha} / H_{\alpha}, \pi_{\alpha \beta}^{\prime}, D\right)$ is an inverse system and that the $\lim _{\leftarrow} G_{\alpha} / H_{\alpha}$ $=G / H$. For $\alpha$ in $D$, we say that $G_{\alpha} / H_{\alpha}$ is admissible if $H^{n}\left(G_{\alpha} / H_{\alpha}\right)$ $\neq\{0\}$ where the dimension of $G_{\alpha} / H_{\alpha}$ is $n$. Associated with each of the homomorphisms $\pi_{\alpha \beta}: G_{\alpha} \rightarrow G_{\beta}$, there are neighborhoods $V_{1}$ of the unit in $G_{\alpha}, V_{2}$ of the unit in the kernel of $\pi_{\alpha \beta}$, and $V_{3}$ of the unit in a local Lie subgroup of $V_{1}$ such that each element $z$ of $V_{1}$ is uniquely and continuously decomposed into a product $x y$ where $x$ is in $V_{2}$ and $y$ is in $V_{3}$. We shall say that $\pi_{\alpha \beta}^{\prime}$, induced by $\pi_{\alpha \beta}$, is admissible if $V_{1}$, $V_{2}$, and $V_{3}$ can be chosen such that $V_{3}$ is a neighborhood of the unit in $H_{2}$. Further, we say that the coset space is admissible if it is the inverse limit of an inverse system, $\left(G_{\alpha} / H_{\alpha}, \pi_{\alpha \beta}^{\prime}, D\right)$, where each factor space and each function $\pi_{\alpha \beta}^{\prime}$ are admissible.

Theorem 2. If $(S, \cdot)$ is a clan and $S$ is an admissible coset space, then $(S, \cdot)$ is a group.

The proof of the above, with the requirement that $S=G / H$ with $H$ connected can be accomplished in the same manner as the proof of Theorem 1. The requirement that $H$ be connected can be removed by using the results of $\mathrm{Hu}$ [5].

We suspect that the hypothesis of Theorem 2 can be weakened to require only that $S$ be a coset space.

Note that Theorem 1 provides a tool for conveniently showing that certain spaces cannot support group structures. For example, let $S$ be the Tychonoff cube and $G$ the circle group. $S$ will support a clan which is not a group. Thus $S \times G$ with the coordinatewise operation is a clan which is not a group. Thus $S \times G$, which is homogeneous and does not have fixed point property, cannot support a group structure. Indeed, the product of a continuum which will support a clan that is not a group with any continuum which will support a clan cannot support a group structure.

\section{REFERENCES}

1. D. R. Brown, Topological semigroups in inverse limit spaces, Master's Thesis, Louisiana State University, 1960.

2. S. Eilenberg and N. Steenrod, Foundations of algebraic topology, Princeton Univ. Press, Princeton, N. J., 1962.

3. H. Hopf, Über die Topologie der Gruppen-Mannigfaltigkeiten und ihre Verallgemeinerungen, Ann. of Math. 42 (1941), 22-52.

4. $\mathrm{S} . \mathrm{T} . \mathrm{Hu}$, Cohomology rings of compact connected groups and their homogeneous spaces, Ann. of Math. 55 (1952), 391-419.

5. - Cohomology relations in principal fiber spaces, Amer. J. Math. 75 (1953), $60-78$. 
6. D. Montgomery and L. Zippin, Topological transformation groups, Interscience, New York, 1955.

7. A. L. Hudson and P. S. Mostert, $A$ finite dimensional homogeneous clan is a group, Ann. of Math. 78 (1963), 41-46.

8. L. Pontrjagin, Topological groups, Princeton Univ. Press, Princeton, N. J., 1939.

9. A. D. Wallace, The structure of topological semigroups, Bull. Amer. Math. Soc. 61 (1955), 95-112.

UNIVERSITY OF KENTUCKY 